\title{
Photometric and Colorimetric Comparison of OLED, NANOSP, and LCD Television
}

Marcelo K. Zuffo

Balázs V. Nagy

Celso S. Kurashima 


\title{
Photometric and Colorimetric Comparison of OLED, NANOSP, and LCD Television
}

\author{
Marcelo K. Zuffo, Member, IEEE, Balázs V. Nagy, and Celso S. Kurashima, Member, IEEE
}

\begin{abstract}
The LCD - Liquid Crystal Display technology is currently the best known of flat screens TVs. However, two innovative and advanced technologies are emerging to the public: the OLED - Organic Lighting Emission Devices, and the NANOSP - Nano Spectrum, also known as Quantum Dot. The aim of this paper is to present a photometric and colorimetric comparison between LCD, OLED and NANOSP. Radiometricspectrum measurements in TVs under test allow a direct comparison of their main characteristics and objectives parameters. Our results bring a detailed understanding of these new technologies performance and their outstanding qualities in the market.
\end{abstract}

Index Terms - TV receivers, HDTV, Photometry

\section{INTRODUCTION}

$\mathrm{T}$ ELEVISION sets using flat-screen monitors are becoming increasingly cheap and popular. Since they make use of microelectronics technologies, flat screens are also increasingly thin and advanced. This feature allow a number of variation such as 3D screens, the curved screens, the ultra-high-definition screens, i.e. $4 \mathrm{~K}$ and $8 \mathrm{~K}$, and the screens of large dimensions. The PDP - Plasma Display Panel technology and the LCD - Liquid Crystal Display technology are currently the most popular in flat screens. However, innovative and advanced technologies are emerging, which are the OLED technology [1] and the Nano Spectrum technology, also known as Quantum Dot [2]. The acronym OLED is the designation for Organic Light Emitting Devices, which is a novel and disruptive technology of flat screens with many advantages in color and contrast response [3][4]. Nano Spectrum is a technology where nanocrystals of semiconductor materials, such as silicon, have quantum mechanical properties. One application of the nano spectrum technology is the manufacture of film that can correct color distortions allowing the construction of LCD-LED flat screens with much better color response [5][6][7]. Both the OLED and the technologies were introduced in the TV world market in 2015 [8]. Thus, considering the diversity of options currently available for flat panel technologies, the comprehension of the OLED and NANOSP features, advantages and disadvantages is desirable. The aim of this paper is to show technology features by a photometric and colorimetric comparison between LCD, OLED and NANOSP television. We have carried out radiometric- spectrum measurements in these three types of TV sets, and we present a direct comparison of their main characteristics and objectives parameters. The results bring a detailed understanding of these new technologies performance and their outstanding qualities in the market. The remaining of this paper presents the related work, the measurement methodology, the results, and the conclusion.

\section{RELATED WORK}

A monitor for computer desktops build with OLED technology was evaluated by Ito et al. [9], and their article reports the technical performance of the display model Sony PVM-2541. The measurements approach is similar to ours in which the OLED monitor is driven by a computer connected to the HDMI input port. So, precise image and video are displayed on the screen. Then, the measurements of luminance, color and spectral characteristics of the display were done by a colorimeter and by a spectral radiometer. The authors present an analysis of these parameters: luminance gradation, additivity of RGB luminance values, color space and spectral distribution, luminance/color uniformity, differences between individual displays, difference in luminance between vertical and horizontal gratings, and temporal characteristics. The conclusion highlights for this particular OLED display are: excellent luminance and color uniformity, wide color space, and rapid luminance rise/fall times.

The work by Luo et al. [10] has a detailed description of the nano spectrum technology for television display construction and the working principle of color enhancement on the display screen. In the last part, the authors briefly discuss the performance of a NANOSP TVs build with QDBPLC (Blue Phase Liquid Crystal, one of the most recent quantum dot technology type) in comparison with OLED TVs. The QD-BPLC TV has advantages in lifetime, power consumption, resolution density, color gamut, and cost. On the other hand, OLED has advantages in true black state, thin profile and flexibility.

Since both technologies of OLED and NANOSP are evolving, the performance reported in related work need to state the television set model. Our evaluation was carried out in the year of 2015 with new television sets by that time. The methodology for the performance evaluation is described in the next section. 


\section{Methodology}

This section describes the procedure of radiometricspectrum measurements in the OLED, NANOSP, and LCD types of TV sets.

The measurements were conducted in a room sealed against entry of light, so that the environment remains completely dark during the measurement procedure.

A computer with a graphics card is connected to the HDMI input of the TV under evaluation. A set of specific videos for measurements are displayed on the TV screen, one at a time, as needed for each measurement. The video image occupies the entire screen area

- The spectroradiometer - CS-1000A device, is placed at approximately $80 \mathrm{~cm}$ apart, in a line perpendicular to the TV screen, as illustrated in Figure 1. This distance is not relevant to the test, but rather the direction to the screen is important to the measurements. Thus, the CS-1000A lens was perpendicularly aimed to one positioning mark on the image used for the ANSI contrast measurement of Fig. 1.

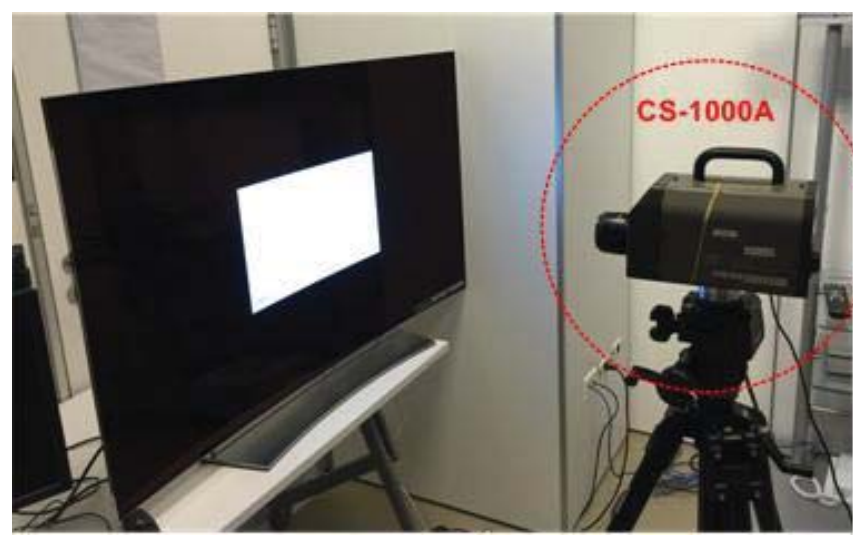

Fig. 1. Position of CS-1000A, perpendicular to an OLED television set

Initially, just after turning on the TV, a video is continuously played for at least 30 minutes before starting the measurements. After this period, measurements are performed within the totally dark environment.

The luminance measurements are carried out with eleven gray images ranging from $100 \%$ to $0 \%$ of the pixel scale in steps of $10 \%$. The image of $100 \%$ grayscale corresponds to the maximum luminance, i.e., totally white. The image of $0 \%$ grayscale corresponds to the minimum luminance, i.e., the black image. The other images are in grayscale.

The TV color emission measurements are performed with images of the three primary colors RGB (Red, Green, and Blue) and the images of the three secondary colors CMY (Cyan, Magenta, and Yellow).

Measurements of the ANSI contrast are conducted with images containing white and black rectangles in a $4 \times 4$ arrangement. With the CS-1000A equipment always in the same position, we measure the emission from white area in the first image, followed by the emission from black area in the second image. In this second measurement, in the black area, the screen does not turn off because there are white areas in the image. The ANSI contrast is the ratio of the luminance of these two measurements.
Additionally, the same ANSI contrast calculation procedure was performed with measurements obtained from the spectrophotometer i1Pro 2. The difference from the above procedure is the position of the equipment. The i1Pro 2 is placed onto the TV screen surface, as illustrated in Fig. 2.

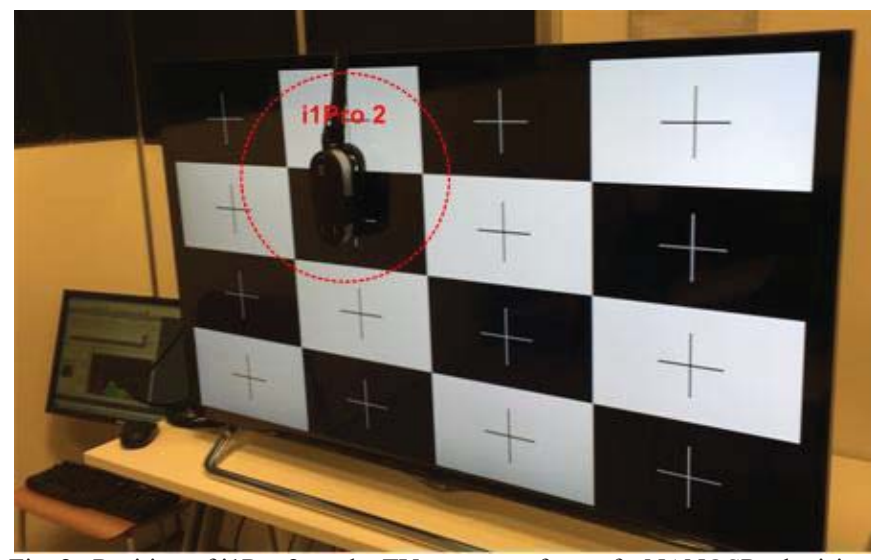

Fig. 2. Position of i1Pro 2 on the TV screen surface, of a NANOSP television set.

In the next step, all the same luminance measurements are performed with room light environment.

The viewing angle measurements were carried out in a dark environment, repositioning the CS-1000A equipment, moving it angularly at a 15 degree angle radius from the previous position, and keeping the lens pointed at the same point of the screen. Thus, it measures the luminance emitted at angles of $15,30,45,60$, and 75 , in relation to the starting position perpendicular to the screen.

Measurements of horizontal luminance uniformity were performed in dark environment, repositioning the CS-1000A equipment, moving it horizontally, but keeping it perpendicular to the TV screen test. The measured screen points are indicated as marks 1, 2, 3, and 4 in Fig. 3 (a). The $100 \%$ white pattern shown in Fig. 3 (b) was used in this measurement. The white area of this image was moved in the TV screen accordingly to the point marks, so that it lies in the center of each measurement position.
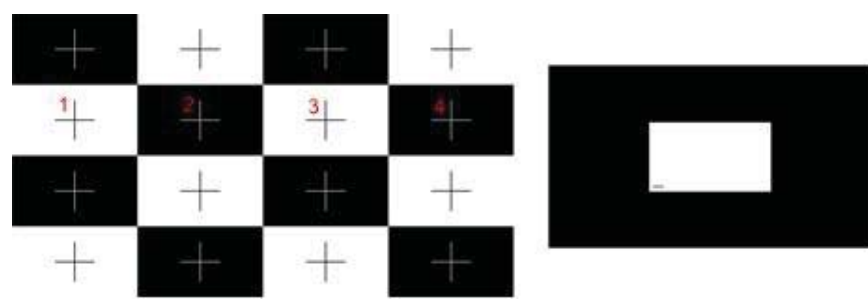

(a) Position marks for the uniformity test

(b) Image of $100 \%$ white

Fig. 3. Points of luminance measures and the image used to perform measurement.

The three evaluated television sets have 55 -inch screen and 4K (3840x2160) pixel resolution. The evaluated models are:

(i) OLED model: 55EG9600-SA,

(ii) NANOSP model: 55UF9500-SA, and

(iii) LCD model: 55UB8500-SA,

All these TV sets are made by LG Electronics.

The radiometric-spectrum measurements with the equipment Konica Minolta CS-1000A, all photometric and 
colorimetric values are based on spectral measurements of radiance in $\mathrm{W} /(\mathrm{m} 2 . \mathrm{sr} . \mathrm{nm})$ in the range of $380 \mathrm{~nm}$ to $780 \mathrm{~nm}$.

Table I presents the television adjustment of parameters settings used on the TVs under test.

TABLE I

TELEVISION ADJUSTMENT PARAMETERS

\begin{tabular}{|c|c|c|c|}
\hline Parameter & OLED & NANOSP & LCD \\
\hline \multicolumn{4}{|l|}{ IMAGE Menu } \\
\hline - Image Mode & $\begin{array}{l}\text { Photo } \\
\text { (User) }\end{array}$ & $\begin{array}{l}\text { Photo } \\
\text { (User) }\end{array}$ & $\begin{array}{l}\text { Photo } \\
\text { (User) }\end{array}$ \\
\hline - Energy Saving & OFF & OFF & OFF \\
\hline \multicolumn{4}{|c|}{ IMAGE MODE Menu } \\
\hline - LED Light & 100 & ---- & ---- \\
\hline - Backlight & ---- & 100 & 100 \\
\hline - Contrast & 100 & 100 & 100 \\
\hline - Brightness & 50 & 50 & 50 \\
\hline - Sharpness & 10 & 10 & 10 \\
\hline - Color & 50 & 50 & 50 \\
\hline - Tone & 0 & 0 & 0 \\
\hline \multicolumn{4}{|c|}{ ADVANCED CONTROL Menu } \\
\hline - Dynamic Contrast & OFF & OFF & OFF \\
\hline - Super Resolution & OFF & OFF & OFF \\
\hline - Color Range & Wide & Wide & Wide \\
\hline - Contour Enhance & OFF & OFF & OFF \\
\hline - Color Filter & OFF & OFF & OFF \\
\hline - Advanced Pattern & OFF & OFF & OFF \\
\hline - Gamma & 2.2 & 2.2 & 2.2 \\
\hline \multicolumn{4}{|c|}{ WHITE BALANCE Menu } \\
\hline - Color Temperature & Medium & Medium & Medium \\
\hline \multicolumn{4}{|l|}{ GENERAL Menu } \\
\hline - Timer & OFF & OFF & OFF \\
\hline - Wait Light & $\mathrm{OFF}$ & OFF & OFF \\
\hline - HD ECO Mode & OFF & OFF & OFF \\
\hline - Software Version & 03.00 .06 & 03.00 .13 & 04.65 .05 \\
\hline
\end{tabular}

\section{RESUlts}

From the measurements of parameters obtained in the previous sections, a comparative analysis was made considering the following parameters shown below:

Luminance: luminance is a measure of the brightness of the white color, in this regard the NANOSP TV showed performance near the OLED TV and far superior to LCD TV;

Angular Luminance: angular luminance is the brightness of the white color seen from a certain angle, in this study we decided to adopt the extent of 45 degree tilt angle. In this case it is observed that NANOSP TV performance is almost equal to the LCD TV, however OLED TV has much higher performance;

Gamma 2.2: the Gamma 2.2 curve is the standard measure of grayscale brightness in flat panel TVs; and the higher adherence to Gamma 2.2 curve the better the contrast of images in grayscale. In these measurements, the OLED TV has almost total adherence to the Gamma 2.2 curve. The LCD TV has median performance and NANOSP TV has lower performance;

Uniformity: this measure evaluates the brightness distribution along the panel. The three TVs have a very great uniformity, and the outstanding uniformity of OLED TV is less than $3 \%$.

Room Light Reflection: the flat screen reflection index at room light is an important parameter to measure the influence of ambient room light on the image. In this aspect the OLED TV showed a superior performance than the LCD TV and NANOSP TV.

Contrast: Contrast measures the difference between white and black levels. The contrast of the OLED TV is far superior to the LCD and the NANOSP TVs.

RGB Interference: the RGB interference measures the degree of spectral overlapping between colors, the smaller the interference of $\mathrm{R}$ color on the $\mathrm{G}$ color, and the interference of $\mathrm{G}$ color on B color, the greater is the separability and fidelity of colors in the screen image. In this aspect the OLED TV showed far superior performance than LCD and NANOSP TVs.

Color Gamut: the color gamut represented in the form of a triangle in the CIE diagram represents all the possible space of colors that can be displayed by a Flat screen. In this aspect the TV OLED presented the color gamut with the largest area, followed by NANOSP TV and the LCD TV.

A comparative radar diagram is shown in Fig. 4, which was constructed by normalization of numerical measurements results. The next subsections discuss the performance results for each of these parameters.

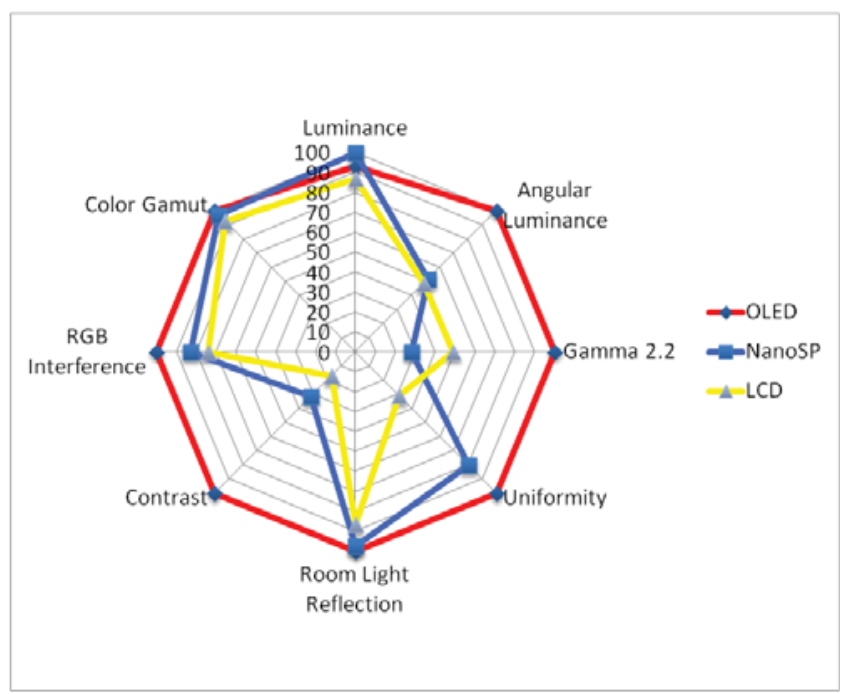

Fig. 4. Radar Diagram of Analyzed Parameters.

\section{A. Maximum luminance}

The maximum luminance measurement was made for the $100 \%$ white image. Fig. 5 presents the absolute measurements. 


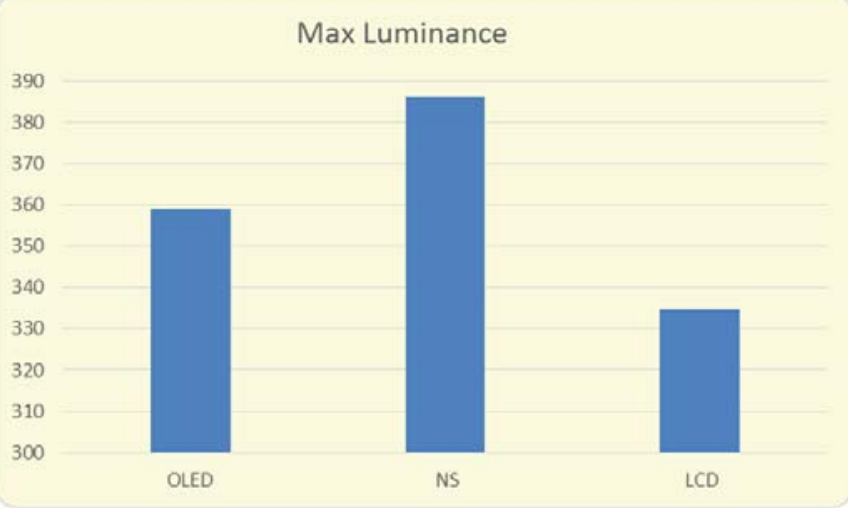

Fig. 5. Maximum luminance results graph.

\section{B. Luminance angular dependence}

The OLED TV showed a significantly lower angle dependence of the luminance with the change in viewing angle, as shown in Fig. 6.

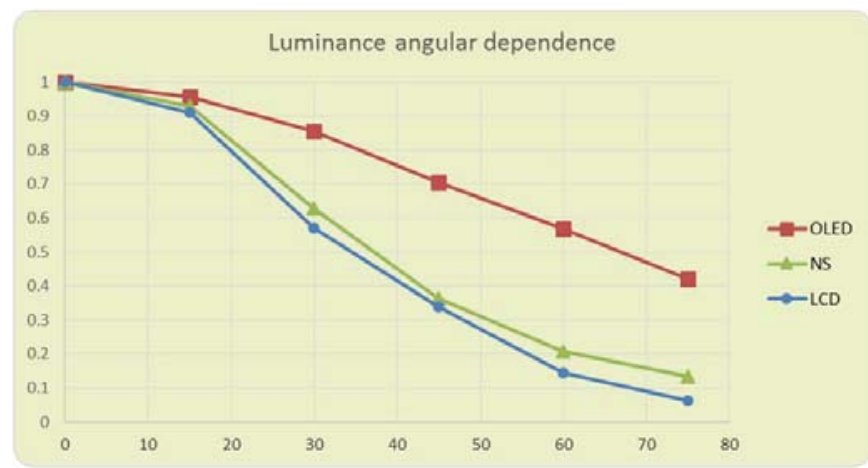

Fig. 6. Luminance angular dependence measures.

\section{Gamma correction: Luminance tracking versus grayscale}

The luminance tracking curve in relation to Grayscale adopted by the industry is the 2.2 Gamma correction curve. The better the TV the closer to Gamma 2.2 function curve. The OLED TV presented the best approximation to the Gamma 2.2 curve, as shown in Fig. 7.

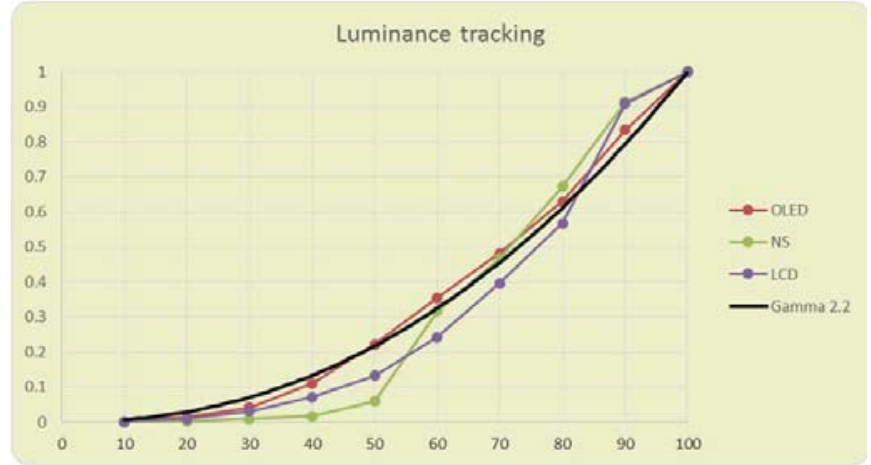

Fig. 7. Luminance tracking graphs.

\section{Horizontal luminance uniformity}

The results of the horizontal luminance uniformity test have few significant differences for all the tested televisions, as shown in Fig. 8.

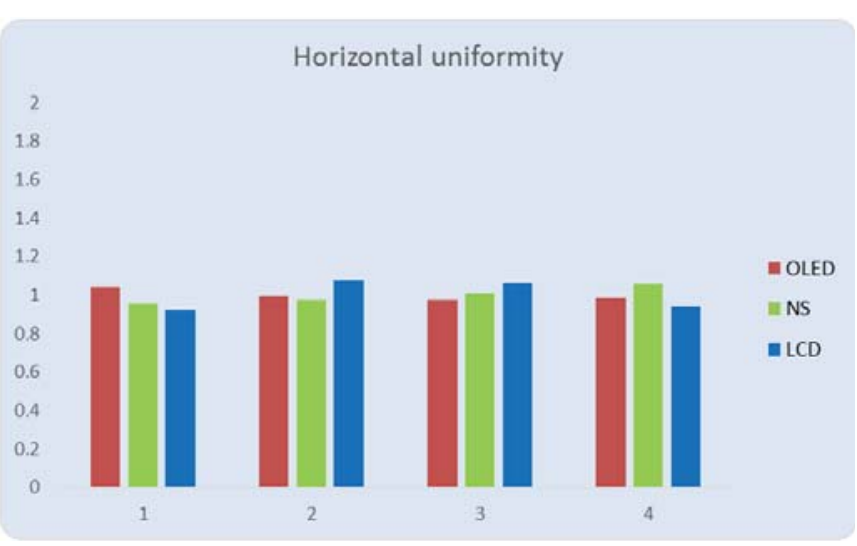

Fig. 8. Results of the horizontal luminance uniformity test.

\section{E. Room lighting reflection effect}

When tested in typical room lighting environment, with the screen turned off, the screen reflection was significantly lower in the case of OLED TV, as shown in Fig. 9.

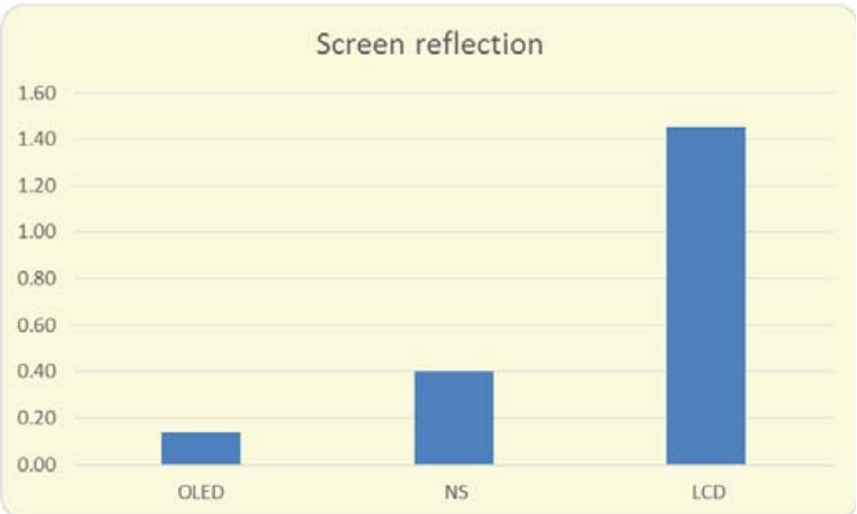

Fig. 9. Screen Reflection with room lighting.

\section{F. ANSI Contrast}

The contrast values, calculated as the ratio between white and black luminance of ANSI standard in the same measuring point were greater on the TV screen OLED as shown in the graph of Fig. 10, with the i1Pro 2.

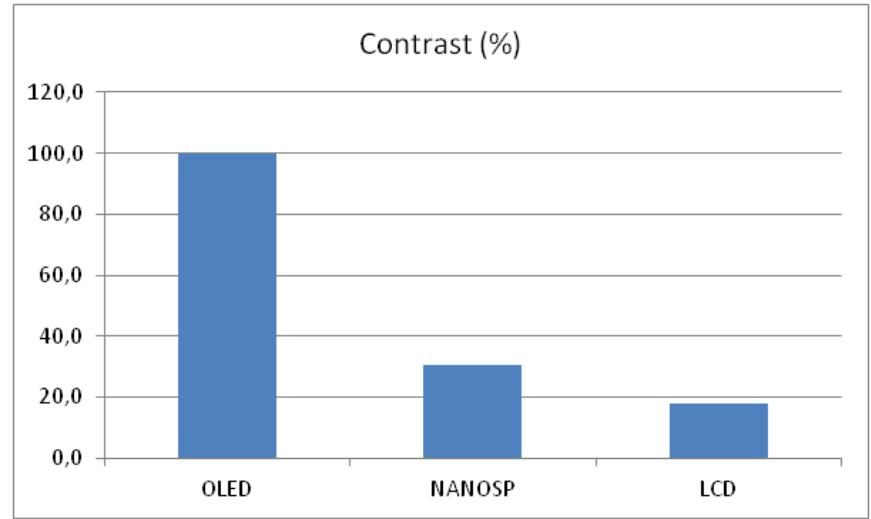

Fig. 10. Contrast ratio of ANSI standard, measured by i1Pro $2 .$. 


\section{G. RGB Spectral crosstalk}

This study shows a calculation of the power interference ratio of each individual channel and the other two channels within each color band. This value is called the signal-tointerference ratio (SIR), and it is calculated in decibels (dB). The higher the value of SIR, the smaller is the spectral interference.

Fig. 11 shows the interference on the G channel for OLED TV set, and the calculated SIR is $12.2 \mathrm{~dB}$. Fig. 12 shows the interference on the G channel for NANOSP TV set, and the calculated SIR is $10.1 \mathrm{~dB}$. Fig. 13 shows the interference on the G channel for LCD TV set, and the calculated SIR is $8.9 \mathrm{~dB}$. Thus, we conclude that OLED TV presents a better performance regarding RGB spectral interference than other two TVs.

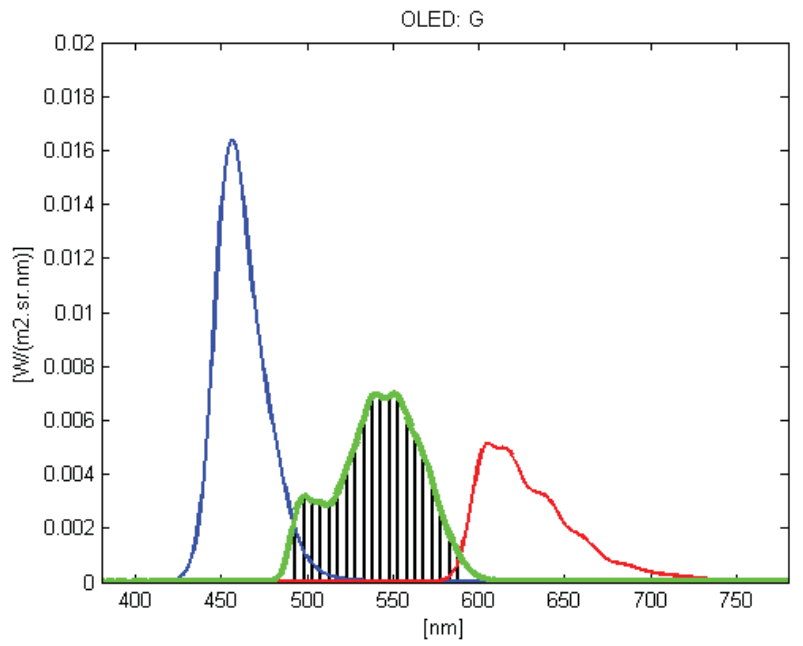

Fig. 11: Interference on G channel for OLED TV.

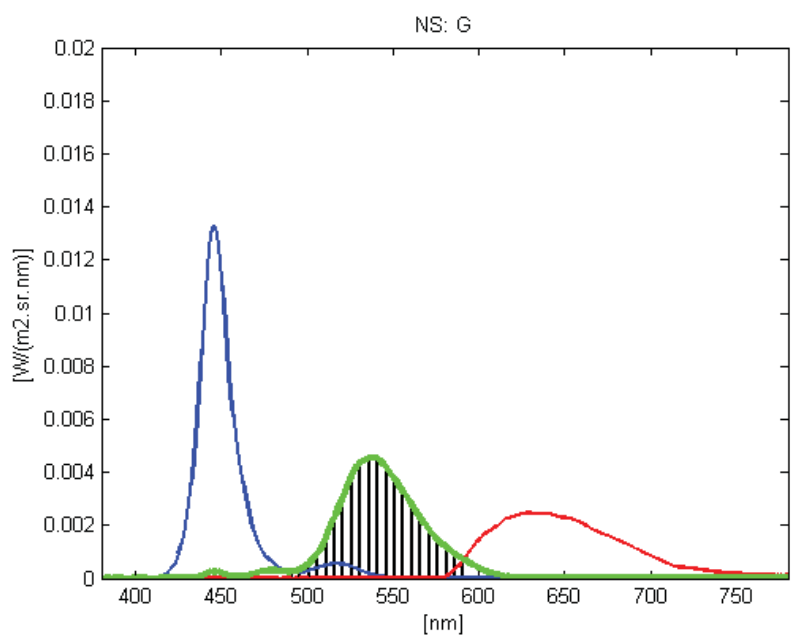

Fig. 12: Interference on G channel for NANOSP TV.

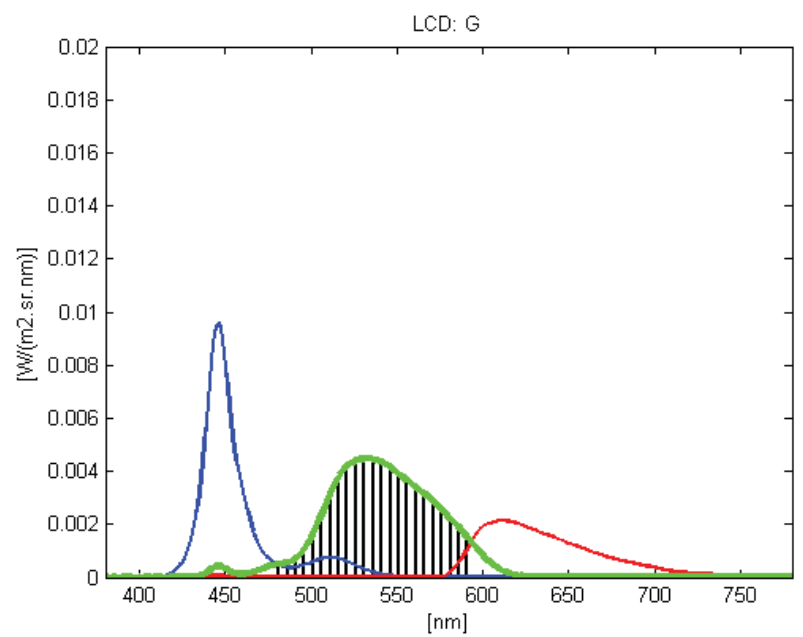

Fig. 13: Interference on G channel for LCD TV.

We also calculated the interference on $\mathrm{R}$ channel and on B channel, and both had similar comparative results. However, the interference on $\mathrm{G}$ channel is stronger in all TVs.

\section{H. Color Gamut}

The OLED TV gamut is larger towards the R-G and G-B axes while the NANOSP TV gamut is larger towards the R$\mathrm{B}$ axis, as shown in Fig. 14.

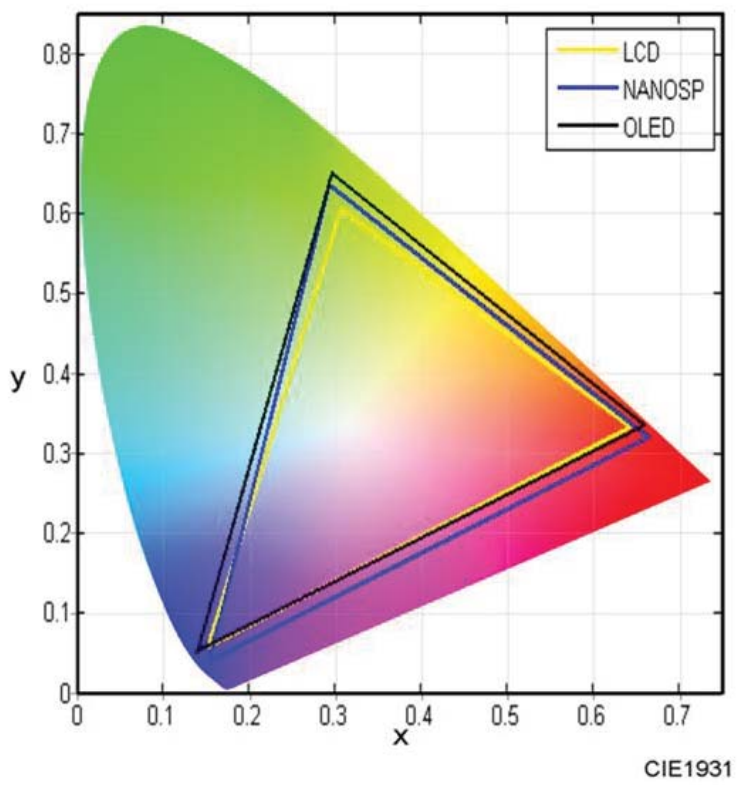

Fig. 14. Comparison of color gamut in the CIE xy diagram.

\section{CONCLUSIONS}

This paper presents a photometric and colorimetric comparison of OLED, NANOSP, and LCD television sets. We have carried out radiometric-spectrum measurements in these three types of TV sets, and we presented a comparison of their main characteristics and objectives parameters. We conclude that the OLED TV showed an overall better photometric and colorimetric performance in all parameters. We also noticed that either OLED and NANOSP 
technologies offer a superior perceived image quality than LCD TVs. In future work we plan to extend the measurements to more parameters, and also we plan to evaluate newer models of television receivers in the market. In regard to television receivers' lifetime estimation, we also plan to carry out measurements in the same models by checking colorimetric and photometric changes after excessive usage time.

\section{ACKNOWLEDGMENTS}

The authors are thankful to LG Electronic - Brazil, for supplying the OLED and NANOSP TV sets, for the technical support, and for the valuable discussions.

\section{REFERENCES}

[1] T.S. Perry, and G. Zorpette, "Oled TV arrives," IEEE Spectrum, vol.50, no.1, pp.46,47, Jan. 2013. doi: 10.1109/MSPEC.2013.6395310

[2] P. Patel, "Quantum dots are behind new displays," IEEE Spectrum, vol.49, no.8, pp.14-17, August 2012. doi: 10.1109/MSPEC.2012.6247547

[3] C. Freudenrich. (2005, March). "How OLEDs Work." [Online]. Available: http://electronics.howstuffworks.com/oled.htm

[4] Liquid Crystals and Photonics Group. "The Basics of Organic Light Emitting Diodes.” [Online]. Available: http://lcp.elis.ugent.be/tutorials/tut_oled

[5] C. Woodford. (2014, November). "What are quantum dots?" [Online]. Available: http://www.explainthatstuff.com/quantum-dots.html

[6] S. Coe-Sullivan, "Enabling Technology: The Virtues of Quantum Dots," Information Display, vol.30, no.3, pp.24-27, May/June 2014 [Online]. Available: http://informationdisplay.org/IDArchive/2014/MayJune/EnablingTec hnologyTheVirtuesQuantum.aspx

[7] J.V. Derlofske, and A. Lathrop. (2014, July). "Advances in Displays: Quantum dot film lets LCDs express $50 \%$ more color." [Online] Available: http://www.laserfocusworld.com/articles/print/volume50/issue-04/features/advances-in-displays-quantum-dot-film-lets-lcdsexpress-50-more-color.html

[8] Tekla Perry. (2015, January). "CES 2015: Placing Bets on the New TV Technologies," IEEE Spectrum. [Online]. Available: http://spectrum.iee. org/tech-talk/consumerelectronics/audiovideo/ces-2015-placing-bets-on-the-new-tvtechnologies

[9] H. Ito, M. Ogawa, and S. Sunaga, "Evaluation of an organic lightemitting diode display for precise visual stimulation," Journal of Vision, vol.13, no.7, pp.1-21, June. 2014. doi:10.1167/13.7.6. [Online]. Available: http://www.journalofvision.org/content/13/7/6

[10] Z. Luo, D. Xu, and S.-T. Wu, "Emerging Quantum-Dots-Enhanced LCDs," Journal of Display Technology, vol.10, no.7, pp.526-539, July 2014. doi: 10.1109/JDT.2014.2325218.

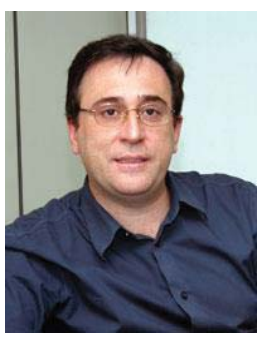

Marcelo Knörich Zuffo (M'88) received the B.Sc. degree, the M.Sc. degree, and the Ph.D. degree in electrical engineering from the University of Sao Paulo (USP), Brazil, in 1989, 1993, and 1997, respectively.

He is Full Professor at the University of Sao Paulo, Brazil, since 2006, at the Department of Electronics Systems. He is also the chief of R\&D activities of the Laboratory for Integrated Systems of USP. Since 2007, he is a Board Member of the Brazilian Digital Television System Forum, where the coordinates the standardization of security for digital television. In 2001, he developed the first Brazilian fully immersive CAVE environment called CAVERNA digital. In 2010, he was General Chair of the ACM International Web3D 2010 Conference held in Los Angeles, CA, USA. In 2000, he was International Resources Chair of ACM SIGGRAPH 2000 held in New Orleans, LA, USA. His research interests include interactive technologies, digital health, high performance computing, virtual reality, graphics computing and visualization.

Dr. Zuffo is a recipient of the 2006's Technology Innovation Prize from the Engineer's Union of Sao Paulo State, Brazil. He was awarded the Medal of Merit Tamandare from the Brazilian Navy, in 2008. Dr. Zuffo is a member of the Brazilian Digital Television System Forum since its foundation in 2007. He is member of ACM SIGGRAPH since 1992.

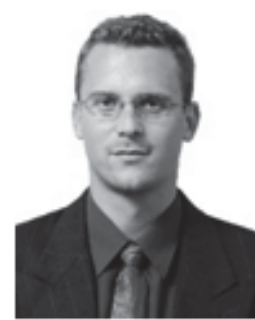

Balázs Vince Nagy graduated as MSc in mechanical engineering at the Budapest University of Technology and Economics (Hungary, 2000), postgraduate degree in economics at the Corvinus University of Budapest (Hungary, 2005), and in illumination engineering at the Budapest Polytechnic (Hungary, 2006). $\mathrm{PhD}$ in mechanical engineering at the Budapest University of Technology and Economics (Hungary, 2009).

$\mathrm{He}$ is senior lecturer at the Department of Mechatronics, Optics and Engineering Informatics at the Budapest University of Technology and Economics and currently is a visiting scientist at the Institute of Psychology of the University of Sao Paulo. His main research fields are visual neuroscience and perception and displays and illuminants as visual stimulators.

Dr. Nagy is an active member of the International Commission on Illumination (CIE) from both Hungarian and Brazilian side and in the International Electrotechnical Commission (IEC) representing Brazil in the maintenance team on Medical image display systems. He also serves as secretary in the medical display related working group of the Brazilian association for normalization (ABNT).

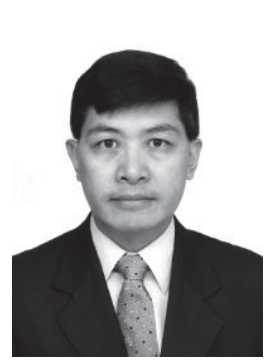

Celso Setsuo Kurashima (M'99) received the B.Sc. degree in electrical engineering from the Universidade Federal de Uberlandia (UFU), Brazil, in 1987, and received the M.Sc. degree, and the Ph.D. degree in electrical engineering from the University of Sao Paulo (USP), Brazil, in 1999, and 2005, respectively.

$\mathrm{He}$ is Associate Professor at the Universidade Federal do ABC (UFABC), Brazil. He joined UFABC in 2006. He was with LSI-TEC, Brazil, as research engineer, from 2003 to 2006. During his doctoral studies, he was Visiting Scholar at the University of North Carolina at Chapel Hill, NC, USA. From 1987 to 1999, he was with NEC Brazil, where he worked as development engineer for over than ten years. He also was a Research Student at the Tokyo Institute of Technology (Titech), Tokyo, Japan. He has experience in PCM circuits, low bit-rate speech coding algorithms, and video coding techniques. His research interests include 
immersive visual communications, interactive 3D video technology, and multimedia signal processing.

Dr. Kurashima is member of ACM SIGGRAPH since 2001 .

Cite this article:

Zuffo, Marcelo K., Nagy, Balázs V. and Kurashima, Celso S.; 2016.

Photometric and Colorimetric Comparison of OLED, NANOSP, and LCD

Television. SET INTERNATIONAL JOURNAL OF BROADCAST

ENGINEERING. ISSN Print: 2446-9246 ISSN Online: 2446-9432. doi:

10.18580/setijbe.2016.6. Web Link: http://dx.doi.org/10.18580/

setijbe. 2016.6 